\title{
Design of Heterogeneous Drive System Based on Object-oriented Devices and Speed Sensor
}

\author{
Wen-Cheng Pu, ${ }^{*}$ Yu-Dian Lin, and Kuei-Hsiang Chao \\ Department of Electrical Engineering, National Chin-Yi University of Technology, \\ No. 57, Sec. 2, Zhongshan Rd, Taiping Dist. Taichung 41170, Taiwan (ROC)
}

(Received March 20, 2019; accepted October 10, 2019)

Keywords: heterogeneous motor, PKM, smart motor, PWM, ATMEGA 328PU

We present a smart motor architecture that incorporates a motor, driver, controller, and communication interface into a single control unit and implement virtualized motor driving. A new pulse width modulation (PWM) technique, called PK modulation (PKM), which is traditionally used as a power supply and is equipped with a communication functionality, is proposed. With this new technique, a multimotor driving system capable of driving heterogeneous motors (such as an AC motor and a DC motor) simultaneously is implemented. The driving system using this smart motor architecture can freely accommodate any change in the number of motors driven, relying solely on the power bus. Other advantages include a simplified driver, effective control of the power signal interference, and reduced number and weight of control lines. In this work, MATLAB/Simulink ${ }^{\circledR}$ is used for signal analysis. MCU ATMEL328PU is chosen as the central controller for hardware implementation. The feasibility of the system architecture is verified as well.

\section{Introduction}

Multimotor driving applications such as a multiaxis moving platform and a robotic arm are becoming popular in the field of automation in recent years. In addition to the issues of mechanism weight reduction and simplification of system architecture and control methods, compatible environments, miniaturization, and smart technologies will be in the highest demand in the future. ${ }^{(1-3)}$ The traditional multimotor driving system has a limit on the number of motors it can drive owing to the constraint of controller performance. When the number of motors to be driven by the controller exceeds the limit, a communication interface, such as the Internet, RS485, or the etherCAD, must be used to achieve coordinated operations. Figure 1(a) shows the multimotor driving application to a robotic arm. Most of the robotic arms have six axes. Multiple motors are used to form multiple robotic arms $\left(\mathrm{R}_{1}-\mathrm{R}_{n}\right)$, which are individually controlled by different controllers (controllers A, B, C, and so forth) via different communication systems and media. As shown in the figure, the robotic arms $\mathrm{R}_{1}, \mathrm{R}_{2}$, and $\mathrm{R}_{n}$ are driven and controlled by controller A. Using current technologies, controller $\mathrm{A}$ is equipped 


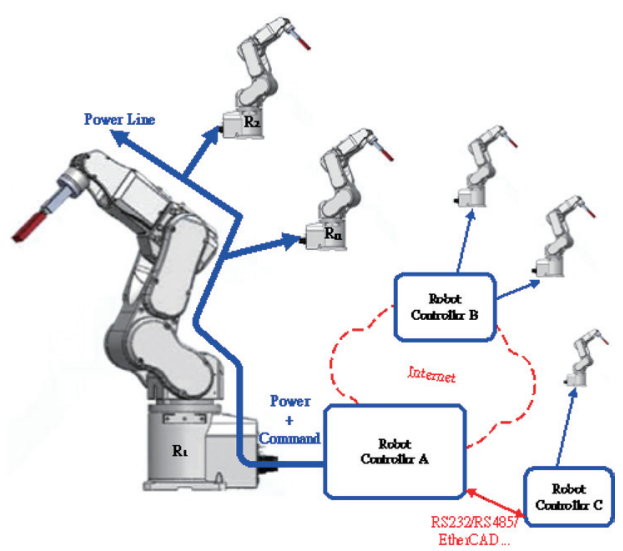

(a)

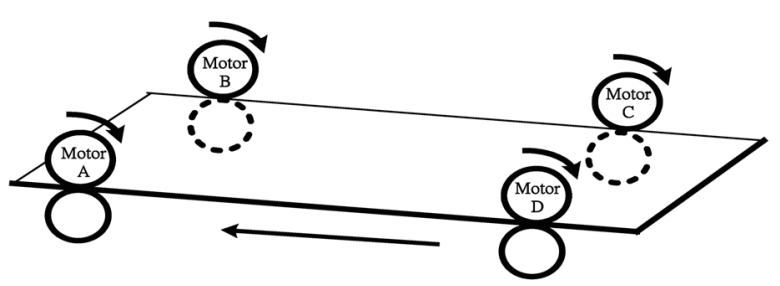

(b)

Fig. 1. (Color online) Architecture of multimotor driving applications: (a) robotic arms, (b) moving platform.

with three control boards, each of them capable of controlling the robotic arm with, at most, six axes. Communication and coordinated control are carried out through a communication interface. Figure 1(b) shows a multiple-axis moving platform (made up of Motors A, B, C, and D) commonly used in glass factories, steel plants, plastic film factories, and LED TV assembly plants to deliver large glass sheets, steel plates, and plastic films. The multiple-axis motors must be controlled and driven synchronously, individually or in groups. Constrained by hardware functionalities and driving methods, traditional multimotor drive controllers are only capable of controlling and driving homogeneous motors (motors of the same type, e.g., all DC or AC motors). ${ }^{(1-5)}$ The so-called AI motor that combines a single DC motor, a driver circuit, and a serial communication interface into a single unit was previously presented. ${ }^{(6)}$ In this design, the power is supplied through a DC bus while commands and data are transmitted using a serial communication interface so that the limit on the number of motors a traditional controller can handle is removed and the control method is simplified. However, the DC bus has a potential problem that the current harmonics can cause interference with the serial communication signals, giving rise to the issue of limited power of the single motor. For the solution, the use of a power line carrier (PLC) communication interface to transmit the DC-brushless-motorrelated data (e.g., current and rotational speed) to a PC at a remote location to achieve remote speed control has been proposed. ${ }^{(7-10)}$ However, a specially designed PLC communication interface is required. The fixed communication channel increases the hardware cost. The signals transmitted over the power line are frequently subject to the interference caused by the filter circuit and ambient magnetic field owing to the properties of the channel. Other than that, the DC motor offers the advantages of stability, controllability, low cost, and the need for only voltage regulation to control the rotation speed of the motor. It is perfect for applications that require high torque. However, the DC motor requires the feedback of the speed signal to control its speed and position. On the other hand, an AC motor has no brushes and therefore does not spark, making it perfect for use in a dusty environment. No additional speed signal is 
needed to perform speed and position control. Even though an additional expensive controller is needed, AC motors are still widely used in various driving applications owing to advantages such as high efficiency, energy saving, and other application features. Motors of various types each have inherent advantages and disadvantages. As shown in Fig. 2, it is hoped that the heterogeneous motors (whether DC or AC motors) incorporated in the control units can be connected by the DC bus. However, because driving strategies are different, heterogeneous motors cannot be controlled and driven at the same time owing to the differences in driving methods. The issues arising in driving heterogeneous motors are therefore seldom addressed in research papers. Driving theories related to both AC and DC motors are introduced below.

\subsection{Three-phase $\mathrm{AC}$ induction motor vector driving principles}

Figure 3 shows a generic motor-driving architecture, in which the voltage of the DC bus is obtained by rectifying the AC power source from the grid and then used to power all units. Let $\dot{x}$ be the state variable and Eq. (1) can descript the dynamic characteristics of the induction motor in the synchronous $d-q$ two-axis rotating reference frame.

$$
\dot{x}=\boldsymbol{A} x+\boldsymbol{B} u,
$$

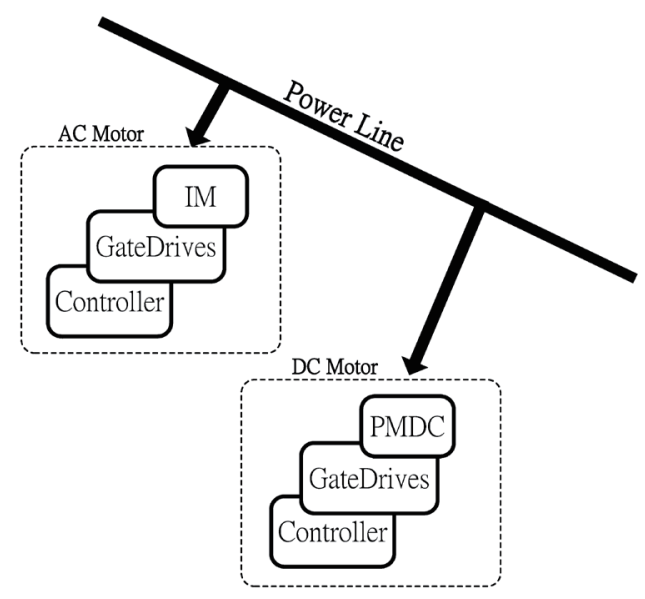

Fig. 2. Concept of heterogeneous motor driving system.

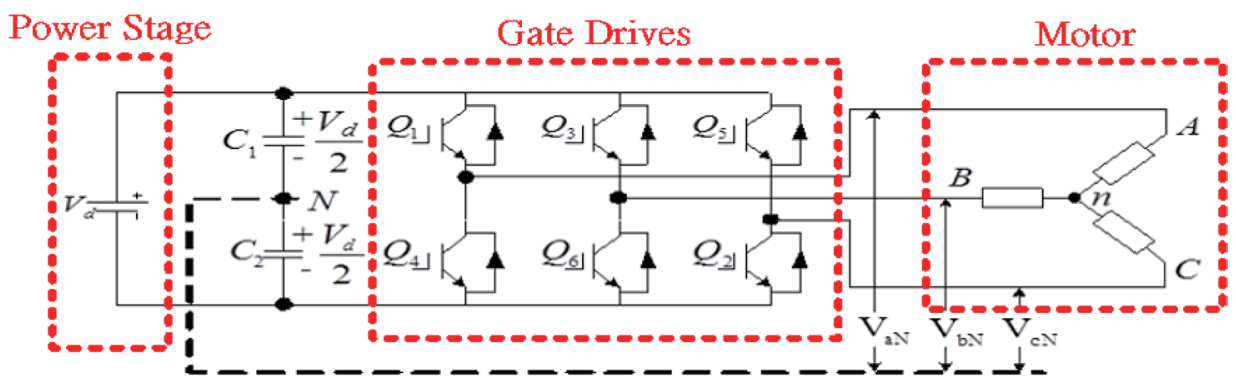

Fig. 3. (Color online) AC motor driver and wiring diagram of three-phase induction motor. 
where

$$
\boldsymbol{A}=\left[\begin{array}{cc}
-\left(\frac{R_{s}}{L_{s}}+\frac{R_{r}}{\sigma L_{r}}\right)-j\left(\omega_{e}-\omega_{r}\right) & \frac{1}{\sigma L_{s}}\left(\frac{R_{r}}{L_{r}}-j \omega_{r}\right) \\
-R_{S} & -j \omega_{e}
\end{array}\right], \boldsymbol{B}=\left[\begin{array}{c}
\frac{1}{\sigma L_{S}} \\
1
\end{array}\right], \boldsymbol{u}=\boldsymbol{v}_{s}^{e},
$$

$\boldsymbol{x}=\left[\boldsymbol{i}_{S}^{e}(t) \boldsymbol{\Phi}_{S}^{e}(t)\right]$ is the state variable, and $\boldsymbol{i}_{S}^{e}(t)$ and $\boldsymbol{\Phi}_{S}^{e}(t)$ denote the stator current and magnetic field, respectively, in the synchronous $d-q$ two-axis rotating reference frame. Then, $\omega_{e}$ represents the synchronous speed of the stator rotation field, $L_{S}$ denotes the stator inductance, $L_{r}, L_{m}, R_{s}, R_{r}, \boldsymbol{v}_{s}^{e}$, and $\omega_{r}$ denote the rotor inductance, mutual inductance, stator resistance, rotor resistance, stator voltage in the synchronous $d-q$ two-axis rotating reference frame, and the speed of the rotor, respectively. Here, $\sigma$ is $1-L_{r} L_{s} / L_{m}{ }^{2}$. Then, the synchronous speed should be estimated by transforming Eqs. (2) to (5) into a stationary reference frame. The superscript " $\Lambda$ " means that the value of the variable is estimated.

$$
\begin{gathered}
\hat{\boldsymbol{\Phi}}_{s}^{s}=\int\left(-R_{s} \boldsymbol{i}_{s}^{s}+\boldsymbol{v}_{s}^{s}\right) d t=\hat{\phi}_{d s}^{s}+j \hat{\phi}_{q s}^{s} \\
\left|\hat{\boldsymbol{\Phi}}_{s}^{s}\right|=\sqrt{\left(\hat{\phi}_{d s}^{s}\right)^{2}+\left(\hat{\phi}_{q s}^{s}\right)^{2}} \\
\hat{\theta}=\tan ^{-1}\left(\frac{\hat{\phi}_{q s}^{s}}{\hat{\phi}_{d s}^{s}}\right) \\
\hat{\omega}_{e}=\frac{d \hat{\theta}}{d t}
\end{gathered}
$$

Here, $\hat{\theta}_{e}$ is the position of the magnetic field; $\hat{\phi}_{d s}^{s}$ and $\hat{\phi}_{q s}^{s}$ are the $d$ - and $q$-axis magnetic components in the static $d-q$ two-axis rotating reference frame, respectively. The position of the magnetic field $\hat{\theta}_{e}$ can also be directly estimated. For stator flux orientation vector control, the speed of the rotor can be both determined with speed sensor hardware and estimated with current sensors. Since the $d$ axis rotates along the reference magnetic field, the $q$-axis rotor flux $q$ is zero. In this manner, the slip speed $\hat{\omega}_{s e}$ and rotor speed can be estimated by transforming Eq. (6).

$$
\hat{\omega}_{r}=\hat{\omega}_{e}-\hat{\omega}_{s e}=\hat{\omega}_{e}-\left(\hat{\omega}_{e}-\hat{\omega}_{r}\right)
$$

The electromagnetic torque $T_{e}$ is modified as in Eq. (7).

$$
T_{e}=\frac{3 P}{4} \operatorname{Im}\left(\boldsymbol{\Phi}_{s}^{e} \boldsymbol{i}_{s}^{e^{*}}\right)=\frac{3 P}{4}\left|\boldsymbol{\Phi}_{s}^{e}\right| \boldsymbol{i}_{q s}^{e}
$$


Here, $i_{d s}^{e}$ and $i_{q s}^{e}$ denote the $d$ - and $q$-axis current components in the synchronous frame, and are used to control the stator flux and electromagnetic torque, respectively. The theory of fluxoriented vector control (FOC) gives the induction motor the torque and flux decoupling control that the DC motor has and the architecture is shown in Fig. 4. The variable-frequency drive controls the induction motor, which is always employed with pulse width modulation (PWM) power signals. However, Eq. (8) is estimated to obtain the slip speed $\hat{\omega}_{\text {se }}$ with only a current sensor used in the sensorless FOC strategy.

$$
\hat{\omega}_{s e}=\frac{L_{s}\left(\tau_{\sigma}^{\prime} \frac{d \hat{i}_{q s}^{e}}{d t}+\hat{i}_{q s}^{e}\right)}{\left(\tau_{r} \hat{\boldsymbol{\Phi}}_{s}^{e}-\tau_{r}^{\prime} L_{s} \boldsymbol{i}_{s}^{e}\right)}
$$

Indeed, an AC motor can be driven by an open loop using a six-step driven scheme that is simpler but less efficient.

\subsection{Driving theory of traditional DC motor}

Figure 5 shows the open-loop four-quadrant driving architecture for a traditional permanent magnet DC (PMDC) motor. In this actual circuit of the driving system, the PWM signal shown in Fig. 6(a) is used to drive the DC motor.

The gate-driven DC motor is shown in Fig. 6(b) and the armature current $i_{a}$ can be obtained from

$$
i_{a}(t)=\Delta i_{a}+I_{a}=\frac{v_{a}}{2 R_{a}}\left[\frac{1+e^{\frac{T}{\tau_{a}}}-e^{\frac{\delta T}{\tau_{a}}}-e^{\frac{(1-\delta) T}{\tau_{a}}}}{e^{\frac{T}{\tau_{a}}}-1}\right]+\frac{v_{a}}{2 R_{a}}\left[\frac{e^{\frac{T}{\tau_{a}}}+e^{\frac{\delta T}{\tau_{a}}}-e^{\frac{(1-\delta) T}{\tau_{a}}}-1}{e^{\frac{T}{\tau_{a}}}-1}\right]-\frac{E}{R_{a}},
$$

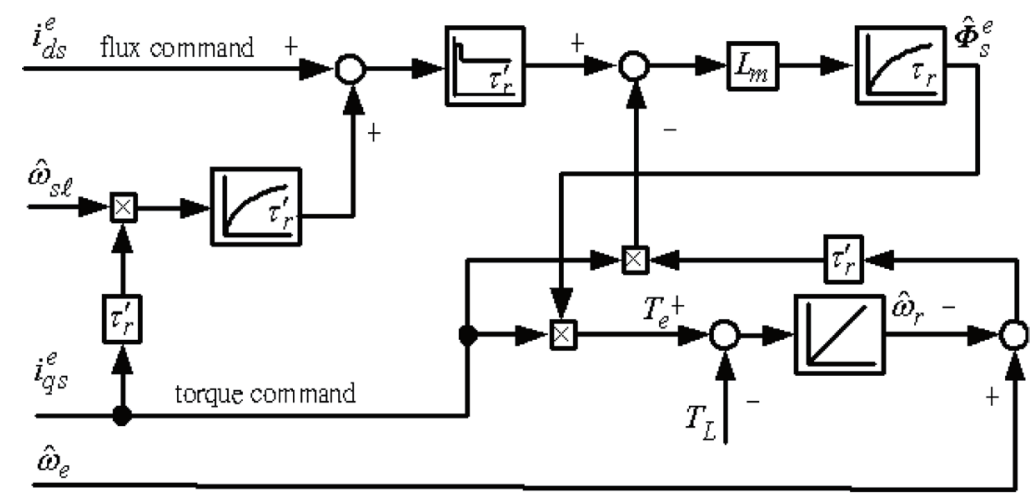

Fig. 4. Control diagram of indirect stator flux orientation of induction motor. 


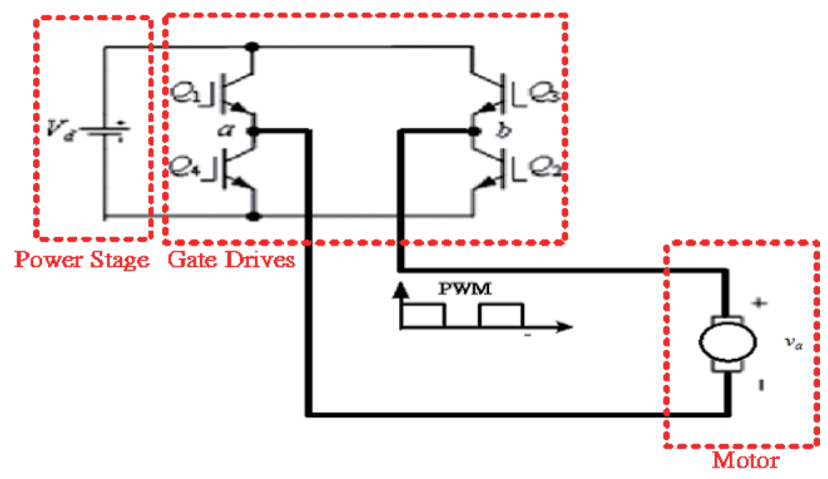

Fig. 5. (Color online) Four-quadrant control system for DC servo motor.

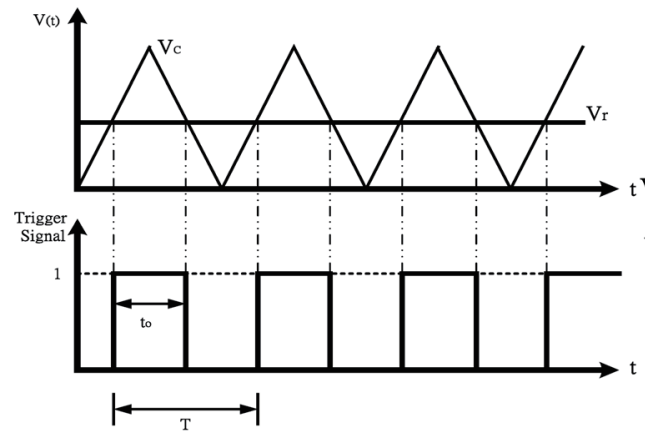

(a)

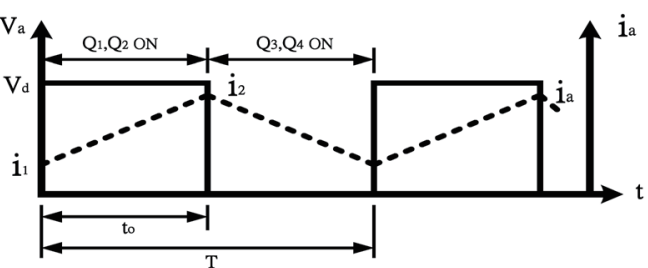

(b)

Fig. 6. PWM cycle operation: (a) PWM signal waveform and (b)PWM current waveform in a cycle.

where $V_{d}, v_{d}, \tau_{a}, R_{a}, T, i_{a}(t), \delta, E, \Delta i_{a}$, and $I_{a}$ are the DC bus voltage, the input voltage, the time constant of the armature circuit, the armature resistance, the modulation period, the armature current, the duty ratio, the inductive voltage in the armature, the $\mathrm{AC}$ component of armature current, and the $\mathrm{DC}$ component of armature current. The perturbing torque $\Delta T_{e}$ calculated from Eq. (10) is a function of $\Delta i_{a}$, which is the cause of vibration and noise. It is known from the equation that the vibration depends mainly on the frequency $f_{c}$ of the carrier Vc. Although choosing a higher $f_{c}$ may reduce the vibration, it increases the switching loss of power switches $^{(11)}$ and reduces the system efficiency. However, the final conclusion is that the PWM power signal is still not equipped with communication capabilities.

$$
\Delta T_{e}=k \phi \Delta i_{a}=\frac{k \phi v_{a}}{2 R_{a}}\left(e^{\frac{1}{2 f_{c} \tau_{a}}}-1\right)
$$

Here, $\phi$ and $f_{c}$ are the magnetic field of the stator and the frequency of the carrier Vc. When the stability is reached, $v_{a}$ can be obtained using Eq. (11) and the electromagnetic torque $T_{e}$ after 
stability can also be calculated using Eq. (12).

$$
\begin{gathered}
v_{a}=\lim _{n \rightarrow 0} \sum_{n=-\infty}^{n=\infty} \delta V_{d} \frac{2 \sin \left(\frac{n \omega_{c} t_{o}}{2}\right)}{n \omega_{c} t_{o}} e^{-j n \omega_{s} t}=\delta V_{d} \\
T_{e}=\lim _{x \rightarrow 0} k \phi I_{a}=k \phi\left(\frac{\delta v_{a}-E}{R_{a}}\right)
\end{gathered}
$$

Figure 7 shows a DC motor driving architecture diagram. In any case, the two driving strategies are obviously different, so they cannot be mixed or operated together in the same multimotor driving platform. However, it can be seen in Figs. 4 and 6 that the PWM signal is used to control the input power whether the DC or induction motor is to be driven. The modulation scheme is employed and shown in Figs. 8(a) and 8(b). According to the theory, the harmonic components of the PWM power signal can be calculated as

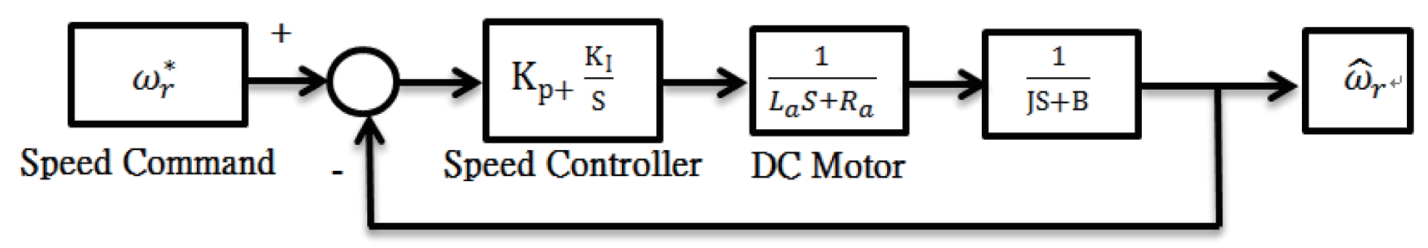

Fig. 7. Structural diagram of DC motor drive.

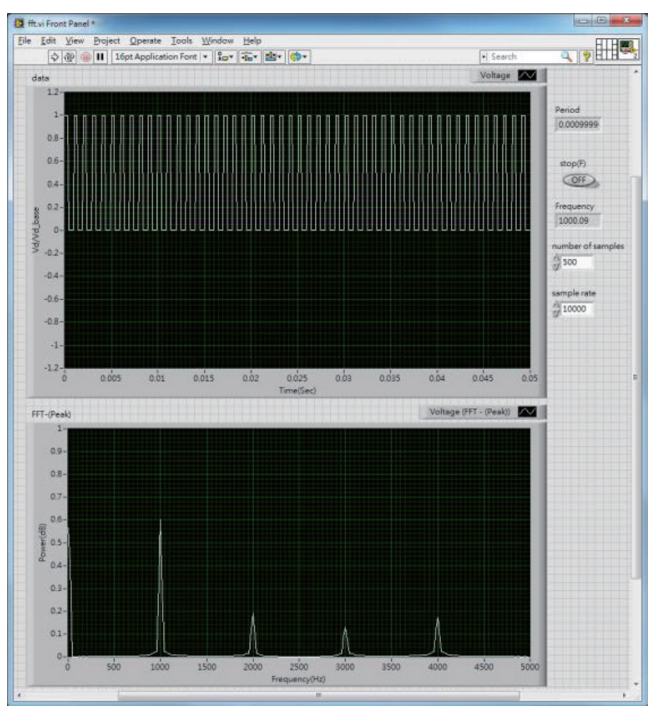

(a)

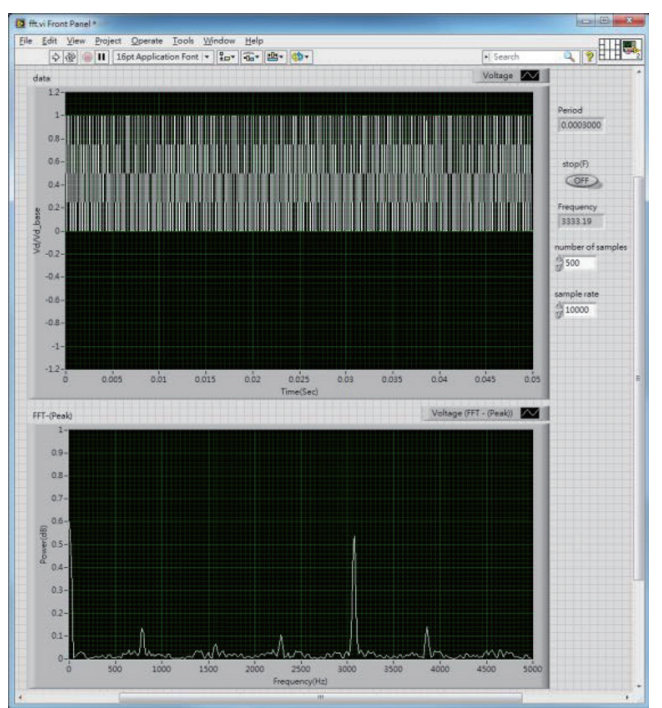

(b)

Fig. 8. (Color online) Frequency spectrum for PWM power signal modulated with carrier frequency $f_{c}$ : (a) 1 and (b) $3 \mathrm{kHz}$. 


$$
v_{m}=\sum_{n=-\infty}^{n=\infty} c_{n} e^{j n \omega_{c} t}, c_{n}=f_{c} \int_{t_{0}}^{t_{0}+\frac{1}{f_{c}}} v_{p}(t) e^{i 2 \pi n f_{c} t} d t
$$

Equation (13) is verified using the rated voltage as the base value, as shown in Figs. 8(a) and 8(b). The spectral analysis of the PWM power signal also reveals that the main harmonics of the PWM power signal are determined by the carrier frequency $f_{c}$. It can be seen that the above principles of the methods used to drive heterogeneous motors are different, but the motors are all driven by PWM power signals. However, PWM power signals have widely distributed harmonics and have no communication capabilities. PWM techniques are commonly used to drive AC and DC motors. One of the most researched topics in recent years is the use of technologies to improve efficiency and eliminate harmonics. References 7 and 11 to 14 are mainly on how to eliminate harmonics by controlling the conduction angle and extinction angle of the power switch. References 11 and 13 are focused on the control of carrier frequency in PWM. This is the simplest and most effective PWM technique, and a simple first-order low-pass filter is used to remove all the harmonics that are multiples of the fundamental frequency. The space vector has also been used ${ }^{(11)}$ to control both the PWM signal and the conduction of power switches in order to eliminate the ramp and improve overall efficiency. References 12 and 13 present a technique for eliminating specific harmonics by calculating and determining appropriate switching angles. However, one disadvantage of this method is that only a small number of specific harmonics can be eliminated at the same time since the calculation consumes excessive resources. The existing papers reveal that the PWM strategy is not equipped with a communication function. In this study, a PK modulation (PKM) technique is therefore proposed to add a communication capability to PWM technology and the control of harmonics. The concept of building a customized motor is also proposed to solve the problems of the limited number of controller axes and the mechanism weight. The wiring and the control method can also be simplified. Above all, the main contribution of this study is the discovery that heterogeneous motors can be driven using the same platform.

\section{Proposed Methods}

To solve the problems mentioned above, in this study project, we present a smart motor architecture and propose the introduction of the object-oriented device (OOD) concept ${ }^{(15)}$ to the smart motor architecture, which combines the controller, gate drive, and communication interface, and incorporates the motor into a single unit. The two smart motor architectures using the OOD concept and incorporating the DC and induction motors are shown in Figs. 9(a) and $9(\mathrm{~b})$, respectively. The purpose is to achieve virtualized motor driving and control. The controller of the smart motor is responsible for receiving commands from the communication interface and driving the motor by the close-loop control method. Figure 10(a) shows the architecture of the smart-motor-based driving platform. To be compatible with the traditional method of driving a motor with PWM power signals, the traditional DC bus Vd circuit must be redesigned, as shown in Fig. 10(a). By adding the front controller, the communication is 


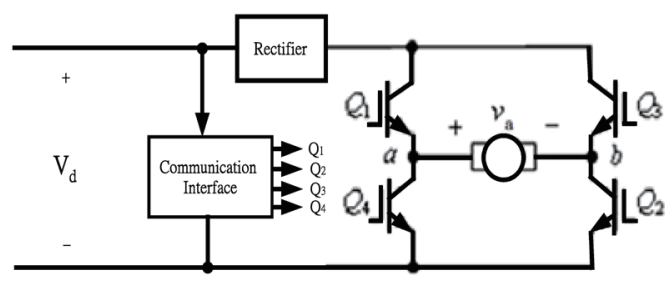

(a)

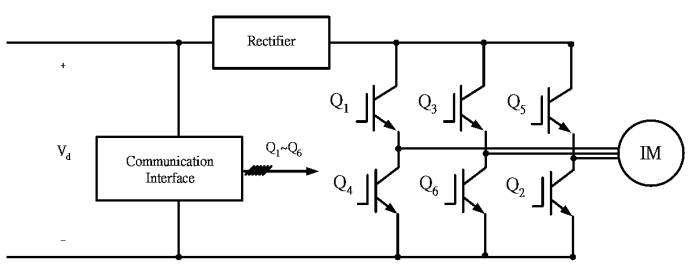

(b)

Fig. 9. Diagram of smart motor architecture with (a) DC and (b) three-phase induction motors.

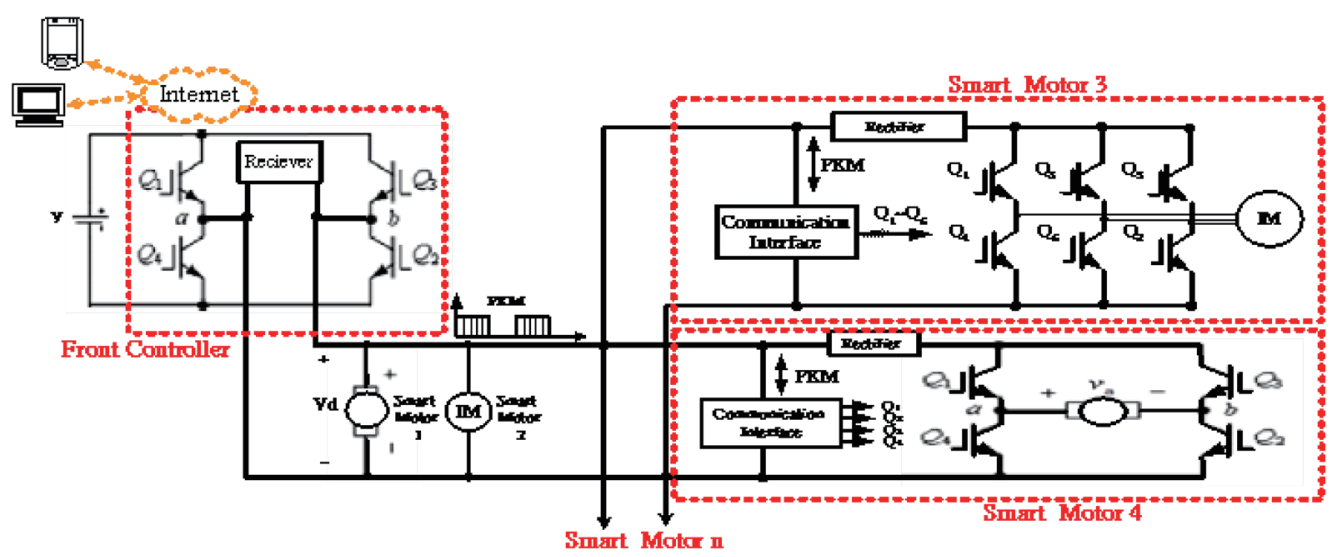

(a)

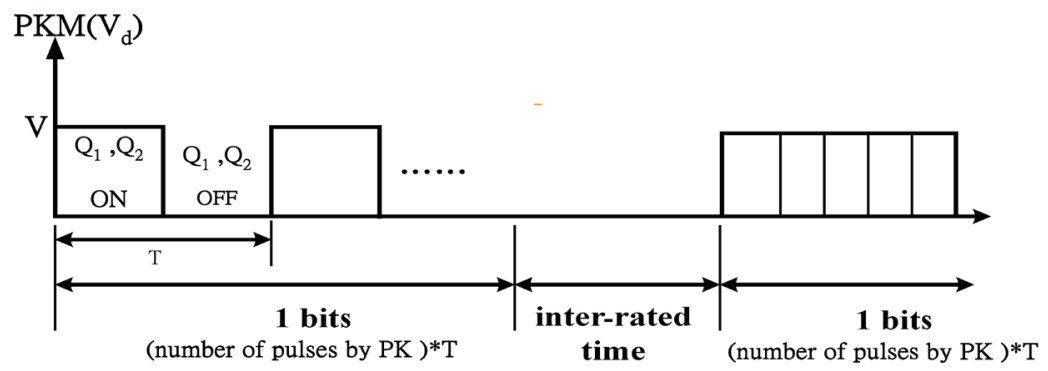

(b)

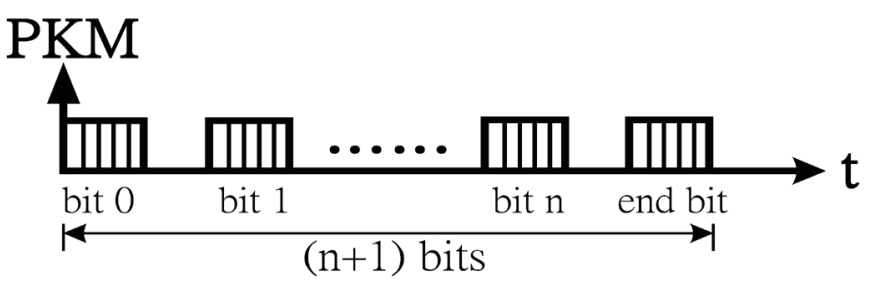

(c)

Fig. 10. (Color online) Smart motor driving system. (a) Diagram of architecture. (b) PKM generator process in RZ signal. (c) Architecture of frame. 
enabled for the PKM method, which is used to modulate the DC output of the power amplifier into the PKM power signal. Basically, the PWM power signal uses the PK encoding scheme to modulate and transmit the data, ${ }^{(15)}$ as shown in Figs. 10(b) and 10(c). The PKM power signal already contains communication data. Therefore, the PKM power signal supplies power to the motor while performing communication at the same time. The motor to be driven, whether it is a DC or AC motor, is powered by the modified DC bus. This new bus design is aimed at replacing the traditional PWM power signal and with one that has a communication capability. Figure 10(a) shows that the smart motors are connected to the newly designed DC bus $\mathrm{V}_{\mathrm{d}}$ circuit. Both return-to-zero (RZ) and not-return-to-zero (NRZ) signal formates can be used. Figures 10 (b) and 10(c) show that the communication interface embedded in the controller is responsible for receiving the PKM power signal in the RZ signal formate, performing PK decoding, and sending the data to the controller. Then, the controller either drives the motor in accordance with the command and internally defined control strategy, or sends the data back. The function of the full-wave rectifier is to convert the PKM power signal into DC current and to power the incorporated motor. With the new DC bus, two power lines are needed to accommodate the free change of the smart motors to be driven. A direct result is that the problems of controlling heterogeneous motors with a single control board and the limited number of motors to be controlled are solved. Driving multiple motors with only two power lines reduces the weight of the power lines required. The wiring complexity is also reduced since the smart motors are connected serially to the DC bus. Furthermore, the PKM power signal carries data and thus reduces the chance of interference with the small signals transmitted over communication interfaces such as RS-232, UART, RS-485, EtherCAD, and PLC. Parameter setting for the communication interface is no longer necessary. The communication speed and data length can be adjusted automatically. Other advantages include improved control of harmonics, reduced interference to the environment, low hardware cost, and good communication quality. In this study, the front controller and PK encoding are used to modulate the output DC voltage V of the power stage into the PKM power signal $v_{p}$. In the communication process, the smart motors need only to count the pulses in order to do PK decoding. The communication process relies on only a series of pulses. ${ }^{(15)}$ Therefore, the PWM power signal $v_{p}$ can be in one of the various forms. The communication speed is also variable. However, a periodic NRZ signal is also used to concentrate the harmonics and avoid interference from other frequencies in the environment. Then, the Fourier analysis of the harmonic components can also be carried out using Eq. (14). The frequency $f_{s}$ of

$$
\begin{gathered}
v_{p}(t)=\sum_{n=-\infty}^{n=\infty} c_{n} e^{j 2 \pi n f_{s} t}=\sum_{n=0}^{n=\infty}\left[a_{n} \cos \left(2 \pi n f_{s} t\right)+b_{n} \sin \left(2 \pi n f_{s} t\right)\right], n \subset N, c_{n}, a_{n}, b_{n} \in R \\
c_{n}=f_{s} \int_{t_{0}}^{t_{0}+\frac{1}{f_{s}}} v_{p}(t) e^{i 2 \pi n f_{s} t} d t \\
a_{n}=f_{s} \int_{t_{0}}^{t_{0}+\frac{1}{f_{s}}} v_{p}(t) \cos \left(2 \pi n f_{s} t\right) d t \\
b_{n}=f_{s} \int_{t_{0}}^{t_{0}+\frac{1}{f_{s}}} v_{p}(t) \sin \left(2 \pi n f_{s} t\right) d t
\end{gathered}
$$


the pulse is used to modulate the signal after PK encoding. The frequency power $S$ of the power signal $v_{p}$ is represented as

$$
\begin{aligned}
& S=f_{s} \int_{t_{0}}^{t_{0}+\frac{1}{f_{s}}} v_{p}^{2}(t) d t=a_{0}^{2}+\sum_{n=1}^{n=\infty} \frac{a_{n}^{2}+b_{n}^{2}}{2}=2 c_{n}^{2}, \\
& \eta=\frac{S_{f_{s}}}{\sum_{n=0}^{n=\infty} S_{n f_{s}}},
\end{aligned}
$$

where $\eta$ denotes the efficiency of the signal after PKM and can be calculated using Eq. (16). Figure 11 shows the spectral distribution of the PKM power signal $v_{p}$ used to transmit " $\mathrm{K}$ " in ASCII code. The DC bus provides the rated voltage as the base value. The frequencies $\left(f_{s}\right)$ of the pulses are 1 and $3 \mathrm{kHz}$. The correctness of the spectral distribution characteristics of Eq. (14) is verified. The direct comparison of the results with those in Fig. 11 reveals that the frequency distribution is more concentrated than that of the PWM power signal. It is known that, while the PKM power signal is used to transmit data, the DC component can be obtained from Eq. (17) when $n=0$. When $v_{p}$ approaches 0 , the $\mathrm{DC}$ loss is

$$
v_{p}=\sum_{n=1,3,5, \infty}\left[\frac{4 v_{d c}}{\pi} \frac{1}{n} \sin \left(2 \pi n f_{s} t\right)\right]=\frac{1}{\pi} \int_{t=-\pi}^{t=\pi} v_{p} d t=c_{0} e^{j 0 \omega t}=0
$$

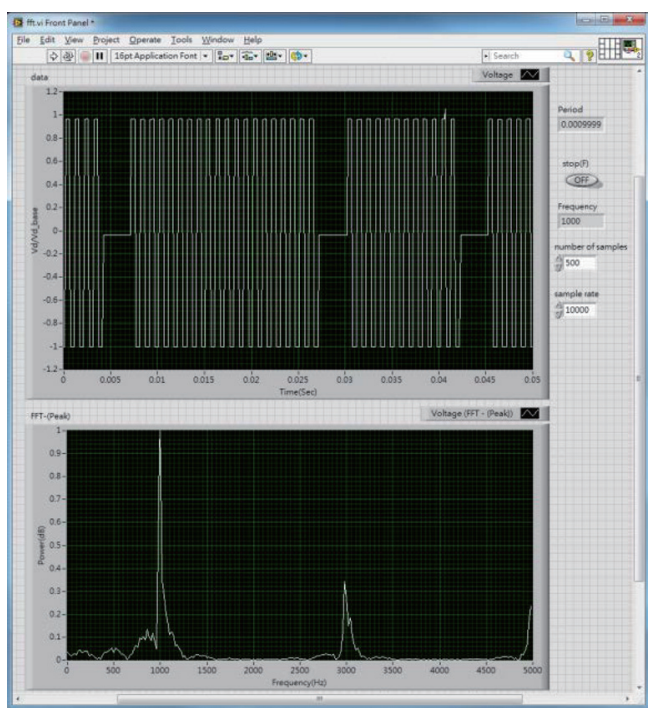

(a)

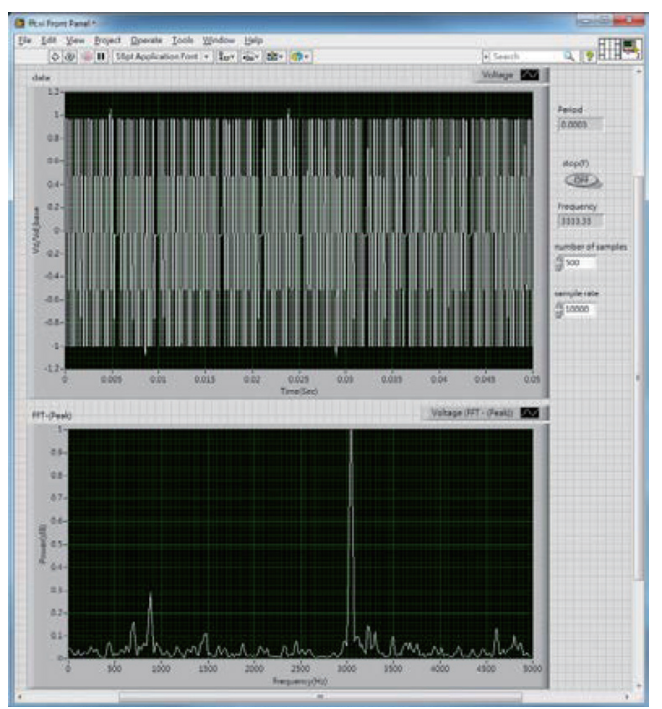

(b)

Fig. 11. (Color online) Frequency spectra of PKM power signals modulated with different $f_{s}$ to transmit "K" ("0100 1011"): (a) $f_{s}=1 \mathrm{kHz}$ and (b) $f_{s}=3 \mathrm{kHz}$. 
reduced and thus the efficiency is improved, as shown in Fig. 11. This also implies that the harmonic distribution of the PKM power signal changes depending on the communication pulse $f_{s}$. However, changing the communication pulse $f_{s}$ does not affect the communication capability. This means that when the PWM power signal is used for communication, the interference to bandwidth caused by the electromagnetic wave in the space can be controlled by changing the communication pulse $f_{s}$ at the transmitting end. By changing the position of the spectral distribution, we can control the source of interference by electromagnetic waves effectively and avoid the frequency bands that easily interfere with the surrounding environment. Therefore, the communication pulse $f_{S}$ can be changed in the implementation to avoid the frequencies that might cause interference to adjust the switching loss of power switches. Although PKM uses $f_{s}$ to modulate communication data, changing $f_{s}$ does not affect the reliability of the communication. ${ }^{(15)}$ This is an outstanding property of PK communication that existing communication technologies do not have. $v_{p}$ can be rectified by the rectifier into a square wave and used as a power supply to smart motors. To achieve the goal of bidirectional communication of smart motors, an identical communication channel of opposite direction for signal feedback must be created within the power stage and central control units. This also implies that each smart motor must have the same rated voltage, which is the only limitation. In addition, since the PWM power signal is digital, no floating-point computation is needed for PK encoding and decoding. A single inexpensive chip is sufficient for encoding and decoding since the demand for computing resources is low. Other advantages of the PK encoding scheme include improved coding/decoding efficiency and low computing/hardware costs.

\section{Measured Results}

To verify the correctness and feasibility of the theories proposed in this study, a hardware platform is implemented, as shown in Fig. 12. The platform adopts the architecture presented in Fig. 10(a) and simulates the multiaxis moving platform shown in Fig. 1(b). Smart motors AD are respectively used in place of respective motors A-D in Fig. 1(b) with speed sensors as servers. Consequently, each smart motor receives only the command and can independently control the monomer itself. This novel and smart motor drive architecture realizes the virtualization of motor operation. In this platform, two smart motors (i.e., three-phase induction motors A and B in Fig. 12) are used to simulate the conditions of no load (motor B, also called ACM2) and a load of $0.1 \mathrm{~N} \cdot \mathrm{m}$ (motor A, also called ACM1). The other two smart motors (i.e., DC motors A and B in Fig. 12) are used to simulate the conditions of no load (motor $\mathrm{C}$, also called DCM2) and a load of $0.1 \mathrm{~N} \cdot \mathrm{m}$ (motor D, also called ACM1). This platform uses a front controller with a Bluetooth communication interface. This interface is used to receive commands from a cellular phone and to transmit data to the phone that is also included in this test platform. The waveform data are sent to the PC via the front controller simultaneously and recorded, displayed, and analyzed using Matlab ${ }^{\circledR} /$ Simulink $^{\circledR}$. The digital oscilloscope is used to display the real-time electrical signals of a single machine. Figures 13(a) and 13(b) show the actual circuits used to drive induction and DC motors, respectively. The tests of single driving, group driving, and synchronous driving are carried out. The four motors of the 


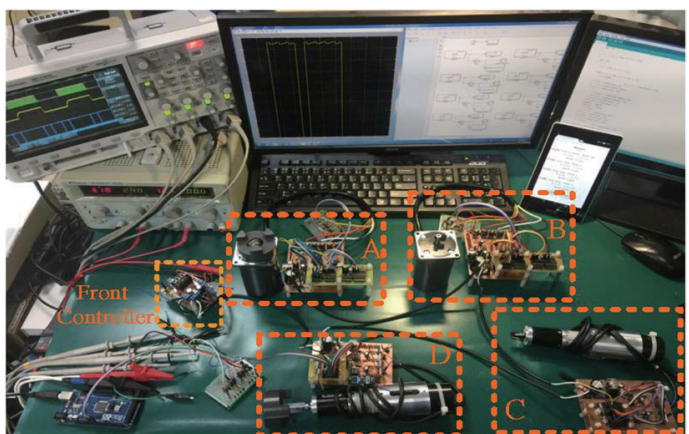

(a)

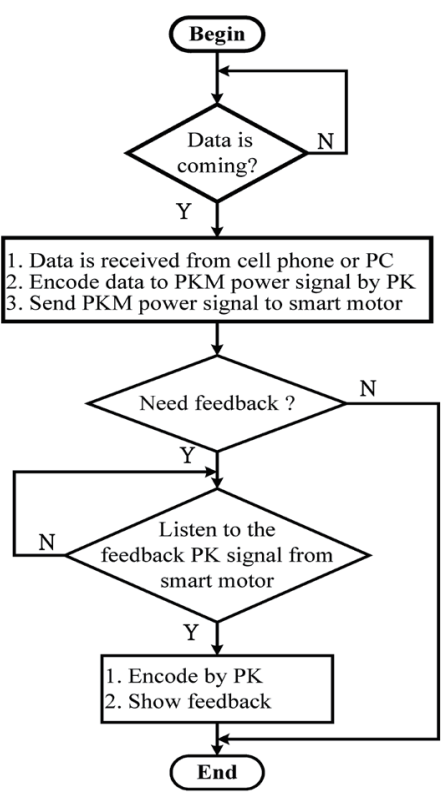

(b)

Fig. 12. (Color online) Smart motor driving platform. (a) Hardware and (b) flow chart of communication.

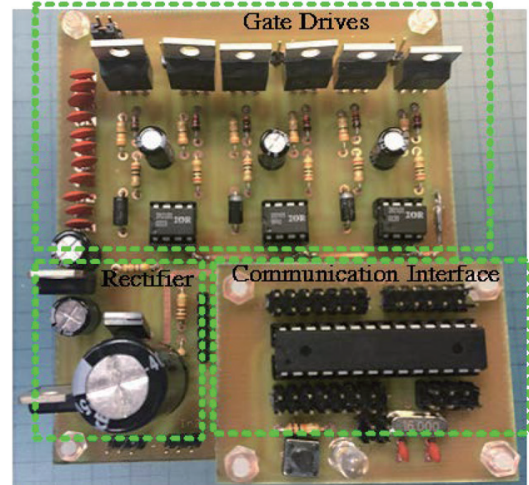

(a)

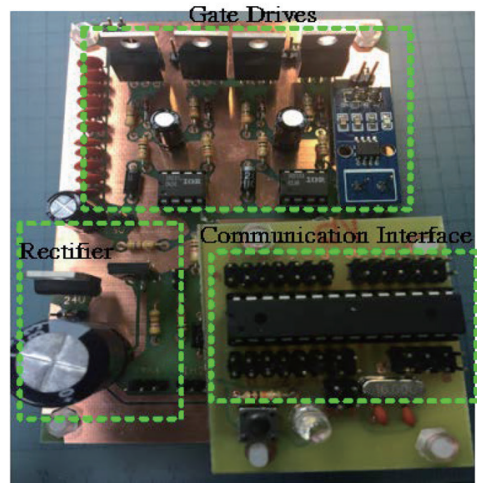

(b)

Fig. 13. (Color online) Smart motor driver circuits. (a) Three-phase induction motor. (b) DC motor.

moving platform are simulated to verify the functions and stability. The output voltage of the power amplifier is controlled at $24 \mathrm{~V}$. To provide a universal voltage $\mathrm{V}_{\mathrm{d}}$ on the $\mathrm{DC}$ bus and the rated voltages to various kinds of motors, a step-down regulator is added to the rectifier in the package circuit. In addition, to be compatible with the traditional driving methods, the format of the PWM power signal is slightly modified so that the output of the PWM power signal after the rectifier still contains the DC value of the power amplifier output.

I. Single-axis drive test: The purpose of this test is to simulate the driving of a single axis of a multiaxis motor driving system for heterogeneous motors. A single-axis $12 \mathrm{~V}$ smart motor (DCM1) and a single-axis $24 \mathrm{~V}$ smart motor (ACM1) are used in this simulation. DCM1 adopts the PWM technique with $f_{c}$ fixed at $200 \mathrm{~Hz}$ and open-loop control. Figure 16 
shows the real-time display of the waveform on the oscilloscope and the data shown on the cellular phone when DCM1 is tested without a load. In Fig. 14(a), the yellow waveform (3) represents the rotational speed. The green waveform (1) represents the PWM signal and the pink waveform (2) is current. The blue waveform (4) is the PKM power signal sent to DCM1 from the front controller. Figure 14(b) shows the operational data received by the cellular phone. Figure 15 shows the driving results when open-loop speed control is used to drive ACM1 by the six-step square-wave control method (with $120^{\circ}$ conduction angle). The PWM technique is also used to drive the motor during conduction. In Fig. 15(a), the yellow waveform (1) represents $i_{U}$. The green waveform (2) is $i_{V}$ and the pink waveform (3) is $i_{W}$. The blue waveform is the PKM power signal.

II. Multiaxis drive test: The purpose of this test is to simulate the simultaneous driving of heterogeneous motors in a multiaxis moving platform. The test is to drive two sets of smart motors, i.e., two $12 \mathrm{~V}$ DCM1 and DCM2, and two $24 \mathrm{~V} \mathrm{ACM1}$ and ACM2 at the same time. $f_{c}$ is fixed at $200 \mathrm{~Hz}$. The first set of smart motors uses open-loop speed control. The second set of motors uses six-step square-wave control (with $120^{\circ}$ conduction angle).

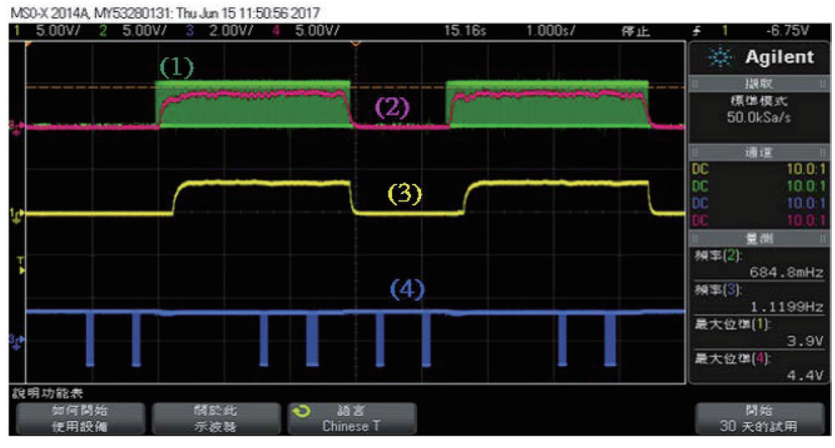

(a)

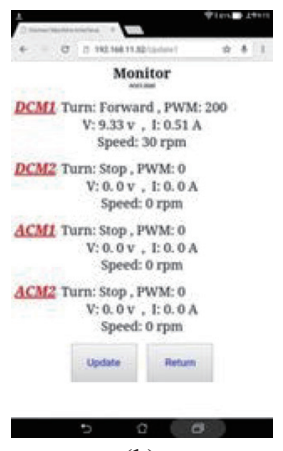

(b)

Fig. 14. (Color online) DCM1 driven without load: (a) waveform and (b) monitoring window on cellular phone showing single-axis drive.

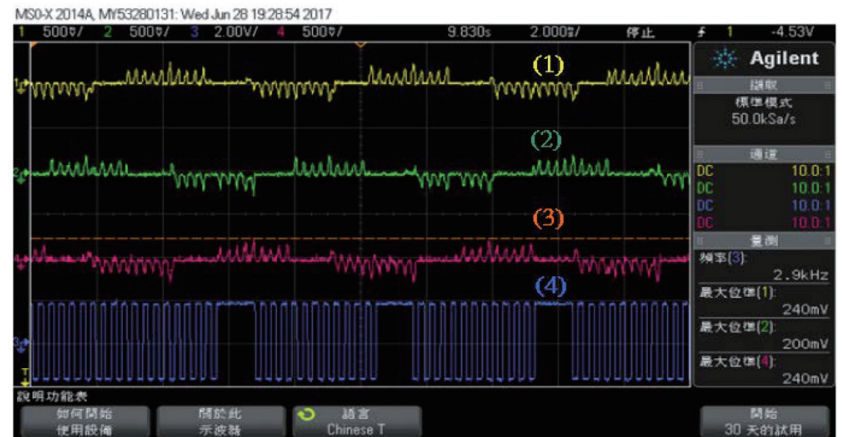

(a)

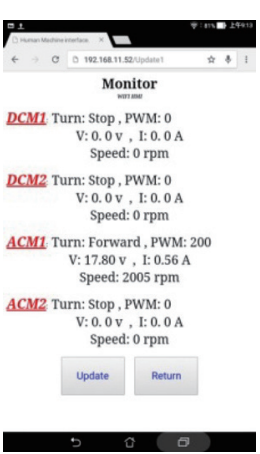

(b)

Fig. 15. (Color online) ACM1 driven by open-loop speed and six-step square-wave control methods, where DCM1 and ACM1 are driven without a load, and DCM2 and ACM2 are driven with a $0.1 \mathrm{~N} \cdot \mathrm{m}$ load. (a) Three-phase current modulated with six-step square wave. (b) Monitoring window on cellular phone showing single-axis drive. 
The PWM technique is also used to drive the motors during conduction. Open-loop speed control is also used. Tests are carried out with and without a load. MATLAB ${ }^{\circledR} /$ Simulink $^{\circledR}$ is used to collect and display smart-motor-related data such as current, formalized voltage, and speed. The data are also collected and displayed using the cellular phone with a bluetooth interface. Figure 16 shows the experimental results of the multiaxis forward rotation of the smart motors. DCM1 and ACM1 are driven without a load. DCM2 and ACM2 are driven with a $0.1 \mathrm{~N} \cdot \mathrm{m}$ load.

III. Multiaxis synchronization: The purpose of this test is to simulate the synchronous driving of multiple motors of the multiaxis moving platform driven by heterogeneous motors. The test is to drive two sets of smart motors, i.e., two $12 \mathrm{~V}$ DCM1 and DCM2, and two $24 \mathrm{~V}$ ACM1 and ACM2 at the same time. ACM1 still uses six-step square-wave control. ACM2 uses stator orientation vector control. Both ACM1 and ACM2 use close-loop speed control. The speed command for DCM1 and DCM2 is $30 \mathrm{rpm}$. The speed command for ACM1 and ACM2 is $2000 \mathrm{rpm}$. MATLAB ${ }^{\circledR} /$ Simulink $^{\circledR}$ is used to collect and display smartmotor-related data. The tests are carried out with and without a load. Data and results are transmitted to and displayed on the cellular phone through the Bluetooth communication interface. Figure 17 shows the experimental results of the multiaxis forward rotation of smart motors. DCM1 and ACM1 are driven without a load. DCM2 and ACM2 are driven with a $0.1 \mathrm{~N} \cdot \mathrm{m}$ load.

The implementation results verify that the smart motor architecture proposed in this study is capable of integrating and driving heterogeneous motors. The monitoring setup and the display of results can be performed remotely. The control method, either close or open loop, can be changed freely in the process, thus simplifying the control process.

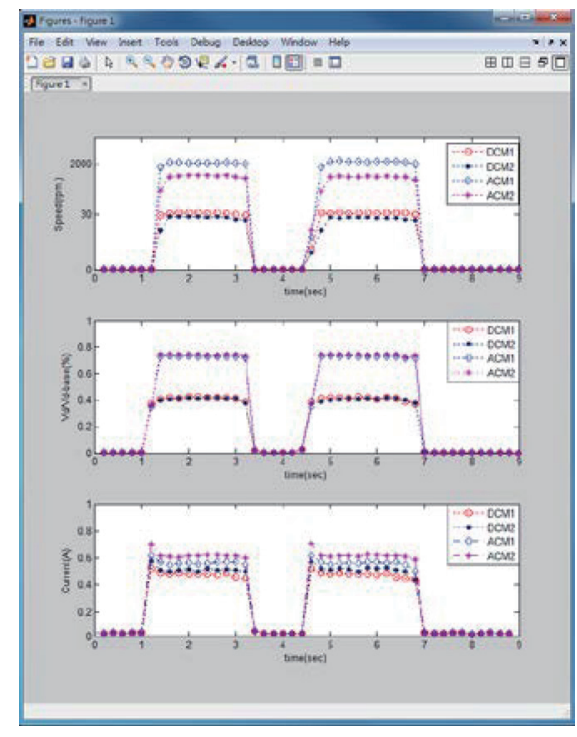

(a)

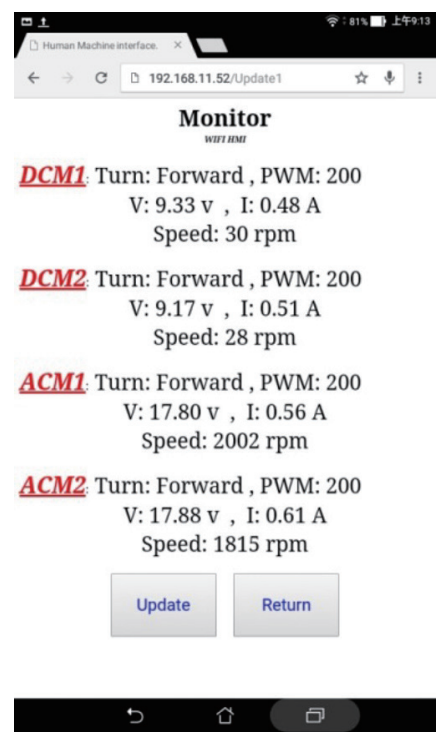

(b)

Fig. 16. (Color online) Four-axis synchronization control of smart motors in which DCM1 and ACM1 are driven without load and DCM2 and ACM2 are driven with $0.1 \mathrm{~N} \cdot \mathrm{m}$ load. (a) Waveforms for the four axes. (b) Monitoring window displaying synchronous forward rotation of multiple axes. 


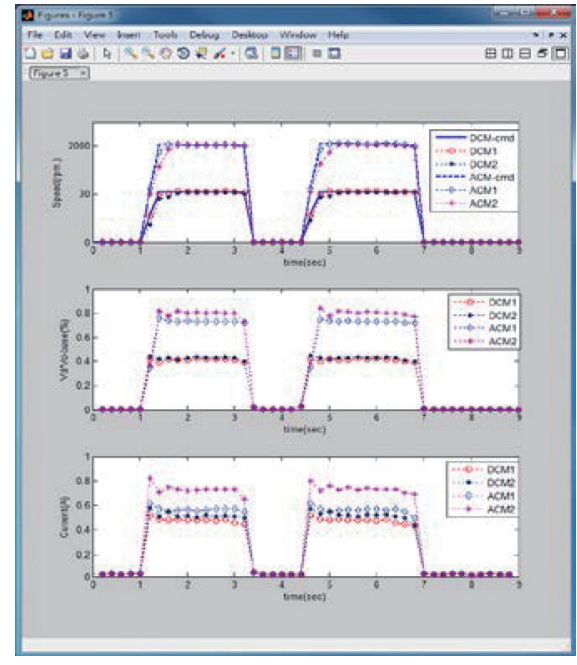

(a)

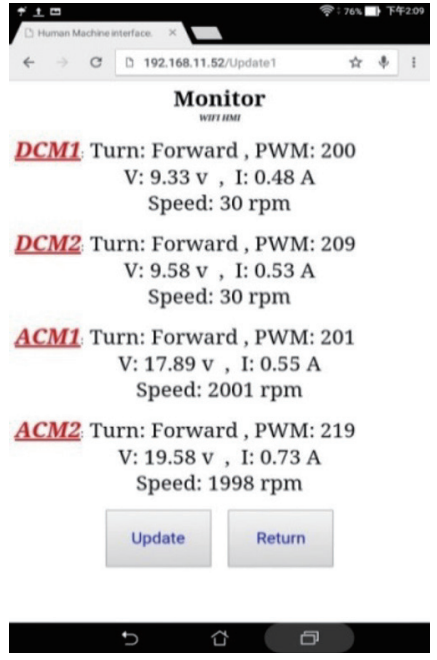

(b)

Fig. 17. (Color online) Multiaxis synchronization control of the smart motors. (a) Waveforms of multiaxis smart motors. (b) Monitoring window on cellular phone showing single-axis synchronous drive.

\section{Conclusions}

Although the multimotor driving system is widely used in various industrial fields, the issues of simplifying the driving process and system architecture are seldom addressed. A smart motor architecture in which multiple motors can be driven with only two power lines is proposed in this study. The system hardware and wiring are greatly simplified. The number of motors to be driven can be changed freely as well. The most valuable contribution of this study is the proposal of a method of sending control and driving commands using a communication channel to drive heterogeneous motors of different power ratings and types. The virtualization concept offers the technology to enable heterogeneous motors to be driven simultaneously. The power lines, used as the power supply and for communication, do not need any expensive or complicated PLC communication interface. Other advantages include a simple architecture, low cost, and high system stability. The most important feature of this architecture is that the communication speed can be changed freely so that the interference caused by harmonics can be avoided. The switching loss of power switches and vibration can both be reduced, which is another major contribution of this study.

\section{Acknowledgments}

This work was supported by the Ministry of Science and Technology, Taiwan, under contract number: MOST 108-2221-E-167-010, duration: August 1, 2019-July 31, 2020. 


\section{References}

1 C. Zhang, M. Niu, J. He, K. Zhao, and H. Wu: Access 5 (2017) 197. https://doi.org/10.1109/ ACCESS.2016.2636224.

2 H. Su, X. Wang, and Z. Lin: Auto. Cont. 54 (2009) 293. https://doi.org/10.1109/TAC.2008.2010897.

3 B. Fidan, V. Gazi, S. Zhai, N. Cen, and E. Karata: Ind. Electron. 60 (2013) 5781. https://doi.org/10.1109/ TIE.2012.2236996.

4 M. Mengoni, A. Tani, L. Zarri, G. Serra, and D. Casadei: Int. App. 48 (2012) 2048. https://doi.org/10.1109/ TIA.2012.2226420.

5 T. Shi, H. Liu, Q. Geng, and C. Xia: Elec. Power App. 10 (2016) 451. https://doi.org/10.1049/iet-epa.2015.0515.

6 G.-M. Chen and Y.-M. Huang: Design of Upper Limb Prosthetics Using AI Motors (Central University Master thesis, Taoyuan, 2006) 1.

7 M. A. Mannah, N. Ginot, and C. Batard: Ind. Elec. 61 (2014) 4238. https://doi.org/10.1109/TIE.2013.2288189.

8 A. Tomar, Y. R. Sood, and D. S. Tomar: Ind. Inform. 11 (2015) 132. https://doi.org/10.1109/TII.2014.2378734.

9 S. L. Stevan, L. d. R. Farias, M. F. C. Barreto, and M. O. Leme: Latin America Trans. 14 (2016) 4241. https:// doi.org/10.1109/TLA.2016.7786300.

10 V. Dégardin, K. Kilani, L. Koné, M. Liénard, and Pierre Degauque: Ind. Elec. 61 (2014) 4816. https://doi.org/ 10.1109/TIE.2013.2293697.

11 T. Boller, J. Holtz, and A. K. Rathore: Ind. App. 48 (2012) 1610. https://doi.org/10.1109/TIA.2012.2210016.

12 G. Poddar and M. K. Sahu: Power Elec. 24 (2009) 1182. https://doi.org/10.1109/TPEL.2009.2013860.

13 A. Mehrizi-Sani and S. Filizadeh: Ind. Elec. 56 (2009) 2894. https://doi.org/10.1109/TIE.2008.2008997.

14 M. A. Mannah, N. Ginot, and C. Batard: Ind. Elec. 61 (2014) 4238. https://doi.org/10.1109/TIE.2013.2288189.

15 W. C. Pu, M. L. Hung, and H. C. Chiung: ICASI2015 1 (2015) 461.

\section{About the Authors}

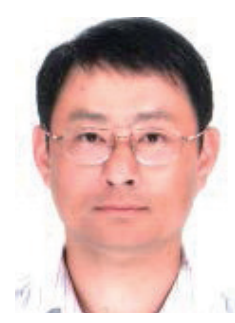

Wen-Cheng Pu received his Ph.D. degree from National Taiwan University of Science and Technology, Taipei, Taiwan, ROC, in 2010. Since 2010 to the present, he is currently an associate professor at the Department of Electrical Engineering, National Chin-Yi University of Technology, Taichung, Taiwan, ROC. His current research interests include the speed estimation of ac motor drives, automatic technology, and the design of microcontroller-based motor drives.

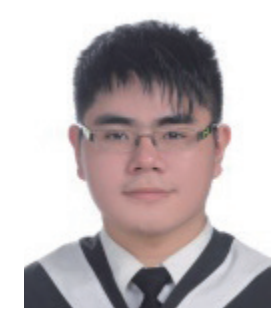

Yu-Dian Lin started master's program in electrical engineering at National Chin-Yi University of Technology, Taiwan, in July 2017. His research mainly focuses on automatic technology and drive technology.

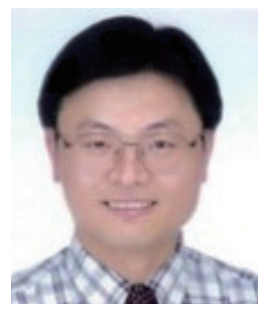

Kuei-Hsiang Chao received his B.S. degree in electrical engineering from National Taiwan Institute of Technology, Taipei, Taiwan, in 1988, and his M.S. and Ph.D. degrees in electrical engineering from National Tsing Hua University, Hsinchu, Taiwan, in 1990 and 2000, respectively. He is presently a professor at National Chin-Yi University of Technology, Taichung, Taiwan. His areas of interest are computer-based control systems, applications of control theory, renewable energy, and power electronics. He is a life member of the Solar Energy and New Energy Association and a member of the IEEE. 